\title{
Spectral Efficiency of Dynamic Licensed Shared Access
}

\author{
Samuel Onidare, Keivan Navaie, Senior Member, IEEE, Qiang Ni, Senior Member, IEEE
}

\begin{abstract}
In licensed shared access (LSA) the radio spectrum is dynamically shared between an incumbent and one or more licensee systems. Protective measures are then applied to the licensees' communication activity to protect normal operation of the incumbent system. Such measures are therefore crucial components of the LSA, and thus fundamentally affect the achievable spectrum efficiency. In this paper we investigate a vertical LSA including an airport traffic control system, as the incumbent, and a mobile network as the licensee. Unlike some previous works that only consider the licensee uplink, we analytically obtain the interference received by the incumbent from the licensee's transmission both in the uplink and downlink. We then obtain optimal uplink and downlink power allocation in the licensee using an optimisation problem with the objective of maximizing licensee's spectral efficiency (SE) subject to the incumbent interference threshold. Furthermore, we investigate the effect of the number of users and cell size on the SE. Our results provide quantitative insights for practical system design and deployment of LSA system. We then examine the whole LSA spectrum utilization by characterising the availability of the LSA spectrum using a tandem queue setting. Using this model we obtain an expression for the spectral utilization as a function of the licensee's achievable spectral efficiency and the statistics of the LSA spectrum availability. Simulation results show more than a seven-fold improvement in the licensee $S E$ using the optimal power allocation. It is also seen that a higher SE gain is achieved with the proposed optimal power allocation in cases where the number of user equipment in the eNodeB coverage area is very small. Furthermore, higher spectrum utilization efficiency is achieved as a result of shorter busy period and higher achievable SE for distant cells.
\end{abstract}

Index Terms-Spectrum efficiency, dynamic licensed shared access, spectrum utilization, interference threshold, tandem queue

\section{INTRODUCTION}

As we enter the zettabytes era of internet data traffic [1], spectrum access approaches must evolve to address the accompanying challenges. In this regard, the licensed shared access (LSA) becomes imperative for spectrum access. In LSA the radio spectrum is dynamically shared between an incumbent and one or more licensee systems. The flexibility in spectrum management provided by the LSA makes it a suitable solution to address the demand for high density machine type communication [2] as well as providing back up solution to unexpected network down time especially for public safety purposes during disaster outbreaks and for rescue operations [3]. Several experimental field trials with live long term evolution (LTE) also suggest that LSA provides a viable

Authors are with the School of Computing and Communications, Lancaster University, Lancaster LA1 4WA, U.K. This research was also partly supported by H2020-MSCA-RISE-2015 ATOM 690750. solution to the challenge of additional spectrum requirement in the sub - $6 \mathrm{GHz} 5 \mathrm{G}$ technology space [4]-[10].

The LSA framework as specified in [11], while ensuring provision of a predictable quality of service (QoS) to nodes on the LSA licensee network, also demands guarantee of protection against harmful interference to the incumbent. Guaranteeing such protection necessitates an exclusion zone which could lead to a significant spatial under-utilization of the spectrum [12], [13]. To overcome this inefficiency, a less restrictive and dynamic exclusion zone becomes necessary. This is more so considering the fact that the initial frequency band proposed for the LSA under the European communication commission (ECC) harmonization, the $2.3 \mathrm{GHz}-2.4 \mathrm{GHz}$, is presently occupied by services such as the aeronautical and terrestrial telemetry [11]. It should also be noted that this frequency band is an initial proposal for harmonization by the ECC and can differ from one country to another depending on the spectrum utilization in each country [14]. Furthermore, the possibility of using the $3.8 \mathrm{GHz}$ has been proposed for the authorised shared access, the concept on which the LSA was based [11].

Considering a scenario, where the incumbent is an airport, and the licensee is a mobile network operator (MNO), telemetry communication between the airport traffic control (ATC) and the flying airplane can only be affected by the licensee transmission within a small portion of the exclusion zone and for a significantly small period of time. As a result, the ATC transmission with the flying aircraft can only experience significant interference from the MNO activities within the flight trajectory if and only if, the telemetry transmission 'radio shadow' radius crosses the MNO cell border [15]. Based on this scenario, the European Union (EU) regulatory framework proposes a dynamic form of LSA [16].

On the basis of this framework, authors in [17], develop algorithms for spectrum allocation in dynamic LSA. It is also shown in [13] that the dynamic LSA can be achieved by either shutting-down licensee's communications or reducing its transmission power to a tolerable level for the incumbent system where and when the incumbent system is active. Simulation results in [13] further indicate that limiting transmission power as in [18] results in SE degradation. An experimental confirmation of the viability of the dynamic LSA is then carried out using a commercial grade LTE testbed in [4]. Similarly, the work in [9], determined the protection criteria for the LSA operation between a MNO licensee and a programme making and special events (PMSE) incumbent system. Experimental demonstrations in [19] further verifies the feasibility of the licensee's compliance with the regulatory requirements of various incumbent systems. 
In [18], a mathematical formulation is provided to analyse applying limiting transmit power policy in dynamic LSA. Using queueing theory and Markov process, [18], [20]-[22] then investigate performance of LSA using metrics such as service interruption and blocking probability, average number of connected users, service failure and mean bit rate. In [19], it is further demonstrated that the radio environment map (REM) can be used to detect the specific area of the incumbent's activity in a dynamic LSA system. In [23], the authors proposed a QoS aware resource allocation and caching for a spectrum sharing between terrestrial satellite system and a cellular network. Different scenarios where the LSA can be deployed for capacity increase in the $5 \mathrm{G}$ technology space as well as a new architecture and enabling technologies were presented in [24]. The work in [25] focuses on the enforcement of compliance by all parties in the LSA to the sharing rules by proposing a framework that discourages licensees from flouting the rules.

As it is seen the common theme of the aforementioned existing works is their focus on the incumbent's protection, although without giving adequate attention to the licensee's network optimization. This is the main motivation of [26] that shows optimizing LSA resources and better spectrum utilization can be achieved while ensuring incumbent's protection from excessive interference with licensee transmit power reduction and antenna downward tilt adjustment. The optimization in [26] focuses on the optimal number of cells that needs to be shut down in order to comply with the interference threshold constraint in the incumbent system within a geographical area. In [27], it is demonstrated that using opportunistic beamforming for resource allocation and scheduling of the licensee system in a LSA setting with horizontal spectrum sharing between two MNOs, the overall throughput of both networks can be improved.

Against this background, in this paper we examine a vertical LSA sharing between an airport traffic control system as the incumbent and a MNO as the licensee. Specifically we focus on improving the licensee's system throughput, where the incumbent is utilizing the spectrum for telemetry services. Unlike some previous works that have only examined the licensee uplink, we consider both the uplink and downlink transmission directions. We begin by an analysis of the interference from the eNodeB to the the ATC tower as a result of the licensee downlink transmission followed by the interference from the user equipment (UEs) to both the ATC tower and the aircraft(s). We then impose the incumbent interference threshold on the licensee system's maximum achievable SE and obtained optimal power allocation for the downlink and uplink transmission. We further examine the effect of various operational parameters that are critical to practical cellular network design and deployment.

Furthermore, we examine the achievable LSA spectrum utilization efficiency within a geographical radius of about $200 \mathrm{~km}$ while employing limited licensee's transmit power to ensure protection against harmful interference to the incumbent system. To do this, we characterize the availability or not of the LSA spectrum as a tandem queue and then derive an expression for the utilization efficiency as a utility function of the achievable SE and the ratio of the busy period of each service layer (eNodeB coverage) to the busy period of all the service layers in the tandem queue. This is more so, considering the fact that the challenge of spectrum scarcity was as a result of inefficiency in the spectrum utilization. Furthermore, in view of the envisaged future capacity demand, we also investigate optimization of the LSA throughput.

The main contributions of this paper are summarised in the following:

- We derive expression for the interference received by the incumbent from the licensee's transmission both in the uplink and downlink. Similar to [13], [18], our previous work in [28], only considers the licensee's uplink in the analysis.

- The effect of various operational parameters (i.e., the number of UEs in licensee coverage area, the eNodeB coverage radius and the adopted eNodeB transmit power) on the SE is also examined. Our results provide quantitative insights for practical system design and deployment of LSA system.

- We introduce a novel performance measure, "decibel capacity gain" to quantify the improvement obtained by the proposed power allocation technique. Using decibel capacity gain we further investigate the SE gain pattern which shed light on the relation of SE to the number of UEs and transmit power adjustment.

- Finally, we propose a novel formulation of the LSA spectrum utilization efficiency as a function of the achievable $\mathrm{SE}$ and busy period ratio of each layer (eNodeB coverage area) to all the service layers (all eNodeB coverage area) within the tandem queue, i.e., the considered geographical radius around the incumbent.

Simulation results show more than a seven fold improvement in the licensee achievable SE compared to a nonoptimized system. It is also seen that a higher comparative SE gain is achieved with the proposed optimal power allocation in cases where the number of user equipment in the eNodeB coverage area is very small. Furthermore, higher spectrum utilization efficiency is achieved as a result of shorter busy period and higher achievable SE for distant eNodeB coverage.

The rest of this paper is organised as follows. In Section II, we present the system model and mathematical formulation. In Section III, we optimise the spectral efficiency of the licensee in both downlink and uplink spectrum, and obtain the optimal power allocation. Then in Section IV we present the spectrum availability model as a tandem queue and define a novel spectrum utilization metric as a function of the optimal SE and average spectrum busy period. Section V, discusses the simulation results and analysis, followed by conclusions in Section VI.

\section{System MODEL}

We consider a circular area with a radius similar to the exclusion zone radius for an airport incumbent. Furthermore, we assume the LSA licensee, a Mobile network Operator (MNO), has multiple cells of radius $\mathrm{R}$ within the considered geographical radius (Fig. 1). The incumbent uses the spectrum 


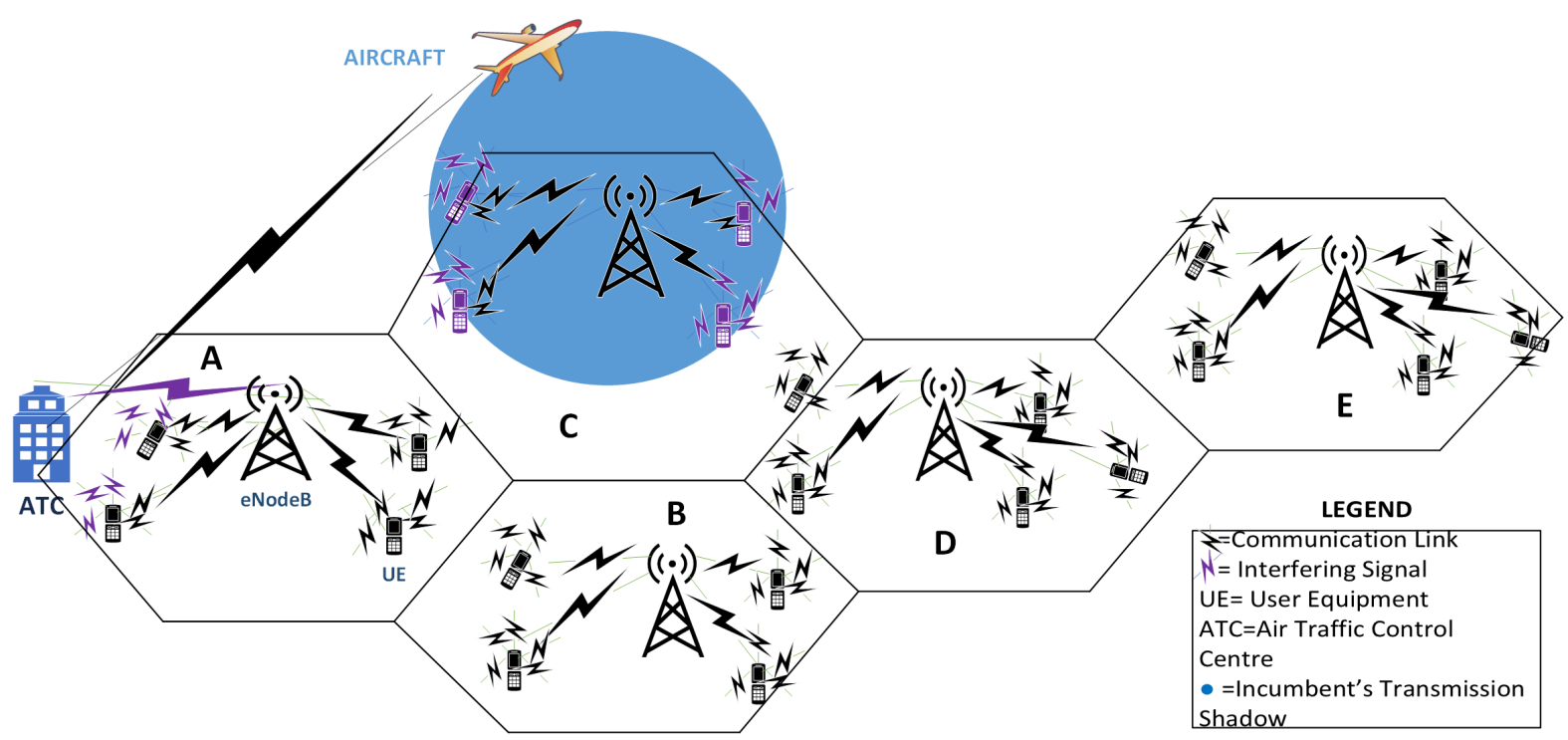

Fig. 1: A schematic of the considered system: Incumbent's transmission shadow only crosses licensee cell C, while cells A, $\mathrm{B}, \mathrm{D}$, and $\mathrm{E}$ are outside the transmission shadow.

specifically when the ATC system is communicating with the aircraft(s). In this time period, the spectrum is considered as busy/unavailable. The rest of the time, the spectrum is referred to as free and available for the MNO unrestricted access.

We further assume a multi-carrier transmission model, e.g., a LTE system with multiple-antenna eNodeB communicating with single-antenna user equipment (UEs), is deployed by the MNO licensee. The channel gain vector for $k$ user equipment is represented as $\boldsymbol{g}_{k}=\left[g_{k 1}, \ldots, g_{k \Lambda}\right]$, where $k=1,2, \ldots, K$, and $\Lambda$ is the number of transmitting antenna. The channel input-output relationship is therefore:

$$
X_{k}=\boldsymbol{g}_{k} \boldsymbol{P}+N
$$

where $X_{k}$ is the channel output, $\boldsymbol{P}=\left[P_{1}, \ldots, P_{\Lambda}\right]$ is the transmit power vector, and $N$ is the complex noise, for the $k^{t h}$ UE. For easy referencing, the symbols used in this paper are given in Table I. Similar to the ATC communication system, the licensee uses a time division duplexing (TDD) system. Furthermore, we also assume that the transmission link from the ATC tower to the aircraft uses the same channel as the MNO uplink transmission and equivalently the reverse link, i.e., from the aircraft to the ATC tower, uses the same channel as the MNO downlink.

\section{A. Incumbent's Received Interference}

In this section, we consider the interference that could impair the ATC transmission to the flying aircraft during the take-off or landing. Here we consider two cases. The first case corresponds to the eNodeB coverage, see cell A in Fig. 1, where the interference to the incumbent's system comprises of rather strong interference signals from eNodeBs and less strong but multiple interfering signals originating from the UEs. We consider the effect of this interference on the ATC tower and the aircraft with the assumption that there is perfect co-operation and synchronization between the licensee and the incumbent operation that ensures no cross- slot interference.

The second case is where the incumbents' interference is only comprised of signals from the UEs. It is reasonable to assume that a critical network design consideration of an LSA licensee is to ensure that the eNodeB antenna height is sufficiently low relative to the ATC tower with a directional pattern (directed downwards to the UEs). Hence, omni-directional transmissions of UEs becomes the main components of the interfering signal [13]. Moreover, for distant licensee cells, i.e., where the ATC tower is outside the eNodeB coverage area, the incumbents received interference only includes UEs' signals within the incumbent's transmission shadow radius. This is shown in eNodeB coverage area C of Fig. 1.

1) Interference from the Licensee $e N o d e B$ Here we assume the licensee's eNodeB is at least of a comparable height and 
TABLE I: List of Parameters

\begin{tabular}{l|l}
\hline Parameter & Description \\
\hline$Q$ & Number of tandem queue service layers \\
$\gamma^{-1}$ & Time between successive flight take-off or landing(s) \\
$\mu^{-1}$ & Time of ATC communication with an aircraft \\
$\tau^{-1}$ & Duration of the spectrum vacancy(s) \\
$s$ & LSA spectrum status \\
$J$ & Number of aircraft landings at or take-offs \\
$k$ & Individual UE \\
$y_{k}$ & Location of individual UE \\
$y_{a}$ & Location of aircraft \\
$n$ & Path loss exponent \\
$\Lambda$ & No of transmitting antenna \\
$P_{k}$ & Transmission power \\
$l$ & Distance related power loss \\
$r$ & Distance between the ATC transceiver and the MNO \\
$g_{d}$ & interferer \\
$v_{d}$ & Ground/horizontal distance between the aircraft and \\
$D$ & a user equipment \\
$I_{\mathrm{th}}$ & Vertical distance/height between the aircraft and a \\
$G$ & user equipment \\
$\boldsymbol{g}_{k}$ & Radius of the interference circular area \\
$\mathbf{K}$ & Incumbent interference threshold \\
$\mathbf{P}$ & Propagation constant \\
$\mathbf{H}$ & Sub-channel gain vector \\
$\eta_{\mathrm{SE}}$ & Sub-channel set \\
$\mathcal{L}$ & transmit power vector \\
$R$ & Channel gain vector \\
$n_{\mathrm{UE}}$ & Lagectrum efficiency \\
\hline & eNodeB cell radius \\
\hline & No. of user equipment \\
\hline & Noisson node distribution density \\
\hline &
\end{tabular}

possibly on the same horizon with the ATC tower. Although careful network design should ensure that the likelihood of this scenario playing out is very low but it is a possibility that is worth considering. Thus, the interference from a licensee's eNodeB located at a distance $r$ to the ATC tower is:

$$
I_{B}=P_{B} h_{B} l(r)
$$

where, $I_{B}$ is the licensee eNodeB interference power, $P_{B}, h_{B}$, is the eNodeB transmit power and fading component respectively, $l(r)$ is the path loss as a result of the separation distance $d$ between the eNodeB and the ATC tower and is given by $l(r)=\|r\|^{-n}, n$ is the path loss exponent. In cases where there are more than one eNodeB within interfering range of the ATC tower, the aggregate interference from multiple eNodeBs is:

$$
I_{\mathrm{AGB}}=10 \log \left[\sum_{B=1}^{N_{B}} I_{B}\right]+30,
$$

where $I_{\mathrm{AGB}}$ is the aggregate interference to the ATC tower from multiple eNodeBs in $\mathrm{dBm}$ (hence adding 30 in the above), $N_{B}$ is the number of interfering eNodeBs, and $I_{B}$ is the individual eNodeB interference power in Watts.
2) Interference from Licensee UE Another, more likely, source of interference to the incumbent system is the omni directional transmission in the uplink direction of the licensee system. The interference from this source comprises of interfering signals from the UEs to both the uplink (airplane receiver) and the downlink (ATC tower receiver) of the incumbent system, in contrast to the interference signal from the eNodeB which only affects the downlink transmission of the aeronautic telemetry system. This is based on the assumption that the eNodeB uses antennas with directional radiation pattern directed downwards to the mobile stations [13]. We consider $K$ UEs, each independently and randomly located at location $x$ within the coverage area of eNodeB. The spatial distribution of the UEs in the coverage area of the eNodeB can then be characterised by a Poisson point process as the following:

$$
\varphi=\left\{x_{1}, x_{2}, \ldots \ldots x_{K}\right\} .
$$

Therefore, the cumulative interference to the incumbent system at a point $y$ as a result of the licensee UEs transmission is:

$$
I_{K}=\sum_{x \in \varphi} P_{k} H_{k} l\left(\left\|y-x_{k}\right\|\right), \quad k=\{1,2, \ldots, K\}
$$

where $P_{k}$, the UE transmission power, is Bernoulli distributed with parameter $p=\operatorname{Pr}\left\{P_{k}=1\right\}$ as the probability that each of the expected $K$ UEs is transmitting, $H_{k}$ the fading coefficient is an exponential random variable. In theory, the MIMO antenna system allows more than one UE transmitting at the same time, but currently in practice, it is still being used primarily for eNodeB simultaneous transmissions to several UEs. Furthermore, the PHY and MAC layers of LTE try to avoid all simultaneous transmissions from UEs connected to a single eNodeB. We can therefore assume that even though there could be many UEs per eNodeB, there is no more than one UE per eNodeB transmitting simultaneously.

We then define distance $\left\|y-x_{k}\right\|,(x \in \varphi)$, as $\|r\| \leq D$, and the intervening area between the UEs is represented as a ball $b(y, D)$, centred at $y$ and of radius $D$. The interference point process $\varphi_{I}=\varphi \cap b(y, D)$ is then defined similar to the inner city model of the Cox process [29], where $\varphi_{I}$, and $\varphi$ are Poisson processes with density $\lambda_{I}$, and $\lambda$, respectively, and $\lambda_{I}=\lambda c_{d} d r^{d-1}$, where, $c_{d}=\|b(0,1)\|$ is the volume of $\mathrm{d}$ dimensional unit radius ball. The probability density function of the interference point process $I_{D}$ is therefore

$$
f_{I_{D}}(i ; \beta)=\frac{1}{\pi i} \sum_{k=1}^{\infty} \frac{\Gamma(\beta k+1)}{k !}\left(\frac{\rho}{i^{\beta}}\right)^{k} \sin k \pi(1-\beta),
$$

where $\beta=\frac{2}{n}, \Gamma($.$) is gamma function, and \rho=\lambda_{I} \pi \Gamma(1-\beta)$.

\section{B. The Interference Propagation Path}

Following the same line of argument as in II-A, we characterize the propagation path for the interference due to the eNodeB as well as the interference due to the UEs. 
TABLE II: Parameters for $\operatorname{Pr}\{\operatorname{LoS}, \theta\}$ in (9).

\begin{tabular}{lccccc}
\hline \hline Environment & $\mathbf{a}$ & $\mathbf{b}$ & $\mathbf{c}$ & $\mathbf{d}$ & $\mathbf{e}$ \\
\hline Suburban & 101.6 & 0 & 0 & 3.25 & 1.241 \\
Urban & 120.0 & 0 & 0 & 24.30 & 1.229 \\
Dense Urban & 187.3 & 0 & 0 & 82.10 & 1.478 \\
Urban High-Rise & 352.0 & -1.37 & -53 & 173.80 & 4.670 \\
\hline \hline
\end{tabular}

1) Air-to- Ground Pathloss Model The path between the UE interferers and the flying aircraft is analogous to the air to ground channel model. According to [30], the transmitterreceiver path/air to ground channel (ATG) can be characterised as:

$$
\mathrm{PL}=\sum_{g} \mathrm{PL}_{g} \operatorname{Pr}\{g, \theta\},
$$

where PL, stands for the path loss between the aircraft and the ground receivers or UEs, $g \in\{\mathrm{LoS}, \mathrm{NLoS}\}$, is the propagation group, where LoS and NLoS are the line of sight and non line of sight propagation respectively. $\operatorname{In}(7), \operatorname{Pr}\{g, \theta\}$ is the probability of LoS and NLoS, $\theta$ is the elevation angle between a ground UE and the aircraft, and

$$
\mathrm{PL}_{g}=\mathrm{FSPL}+\xi_{g} .
$$

In (8) FSPL is the free space path loss and $\xi$ is the excessive path loss, which is, propagation group (LoS or NLoS) and environment dependent. Note that $\xi_{\mathrm{LoS}}$, can be approximated by a log-normal distributed with location variability parameter $\zeta_{\text {LoS }}$ [31], while for the $\xi_{\text {nLoS }}$, an additional building roof top diffraction loss $l_{b}$ [32] is factored into the equation, i.e., $\xi_{\mathrm{nLoS}}=\zeta_{\mathrm{nLoS}}+l_{b}$.

Using the ITU-R recommendations P-1410 [33], $\operatorname{Pr}\{g, \theta\}$ for LoS propagation is obtained in [32] as:

$$
\operatorname{Pr}\{\operatorname{LoS}, \theta\}=a-\frac{a-b}{1+\left(\frac{\theta-c}{d}\right)^{e}},
$$

where $a, b, c, d$, and $e$, are parameters obtained from extensive simulations and presented in ITU-R recommendations P-140 [33] and experimentally validated in [32]. Table II presents the values obtained from the experiment.

Substituting FSPL, (8),(9) into (7) and noting that transmitter-to-receiver distance is $r=\sqrt{v_{d}^{2}+g_{d}^{2}}$, we then express the path loss as a function of elevation angle:

$$
\begin{aligned}
\mathrm{PL}(\theta)=20 \log \left(g_{d}\right) & +10 \log \left(1+(\tan \theta)^{2}\right)+k+\zeta_{\mathrm{nLoS}}+l_{b} \\
& +A\left[a-\frac{a-b}{1+\left(\frac{\theta-c}{d}\right)^{e}}\right], \quad \text { if } \quad g_{d}>v_{d}
\end{aligned}
$$

$$
\begin{aligned}
\mathrm{PL}(\theta)=20 \log \left(v_{d}\right)+ & 10 \log \left(1+\frac{1}{(\tan \theta)^{2}}\right)+k+\zeta_{\mathrm{nLoS}}+l_{b} \\
+ & A\left[a-\frac{a-b}{1+\left(\frac{\theta-c}{d}\right)^{e}}\right], \quad \text { if } \quad g_{d} \leq v_{d},
\end{aligned}
$$

or as a function of the aircraft altitude and the ground/horizontal distance of the UEs:

$$
\begin{aligned}
\operatorname{PL}\left(v_{d}, g_{d}\right) & =20 \log \left(g_{d}\right)+10 \log \left(1+\frac{v_{d}^{2}}{g_{d}^{2}}\right)+k+\zeta_{\mathrm{nLoS}}+l_{b} \\
& +A\left[a-\frac{a-b}{1+\left(\frac{\tan ^{-1}\left(\frac{v_{d}}{g_{d}}\right)-c}{d}\right)}\right], \quad \text { if } \quad g_{d}>v_{d},
\end{aligned}
$$

$$
\begin{aligned}
\operatorname{PL}\left(v_{d}, g_{d}\right) & =20 \log \left(v_{d}\right)+10 \log \left(1+\frac{g_{d}^{2}}{v_{d}^{2}}\right)+k+\zeta_{\mathrm{nLoS}}+l_{b} \\
& +A\left[a-\frac{a-b}{1+\left(\frac{\tan ^{-1}\left(\frac{v_{d}}{g_{d}}\right)-c}{d}\right)^{e}}\right], \quad \text { if } \quad g_{d} \leq v_{d},
\end{aligned}
$$

where $g_{d}$ is the horizontal distance of the UEs in $\mathrm{km}, v_{d}$, is the altitude of the aircraft also in $\mathrm{km}, k=20 \log (f)+92.4$, $f$ is the carrier frequency in $\mathrm{GHz}$, and $A=\xi_{\mathrm{LoS}}-\xi_{\mathrm{nLoS}}$.

2) Extended Hata Model In line with the recommendation contained in the report by the U. S. department of commerce, National Telecommunication and Information Administration (NTIA) in [34], we use the extended Hata model (eHata) for the signal attenuation along the path between the licensee eNodeB and the ATC tower as well as the propagation path between the UEs and the ATC tower. The model is valid for frequency range from $1500-3000 \mathrm{MHz}$, distance of 1-100 $\mathrm{km}$, transmitter and receiver height of $30-200 \mathrm{~m}$ and 1-10 $\mathrm{m}$ respectively. Therefore, the eHata point to point median basic transmission loss for an urban outdoor environment is:

$$
\begin{aligned}
& \mathrm{PL}_{\mathrm{eH}}\left(f, r, h_{B}, h_{R}\right) \\
& \quad=L_{\mathrm{bm}}\left(f, R_{\mathrm{bp}}\right)+10 n \log \left(\frac{r}{R_{\mathrm{bp}}}\right)+13.82 \log \left(\frac{200}{h_{B}}\right) \\
& \quad+v(3)-v\left(h_{R}\right)+\mathrm{PL}_{\mathrm{fs}}\left(f, R\left(r, h_{B}, h_{R}\right)\right) \\
& \quad L_{\mathrm{bm}}\left(f, R_{\mathrm{bp}}\right)=30.52-16.81 \log f \\
& +4.45(\log f)^{2}+\left(24.9-6.55 \log h_{B}\right) \log R_{\mathrm{bp}} \\
& v\left(h_{R}\right)=(1.1 \log f-0.7) h_{R}-1.56 \log f+0.8 \\
& R\left(r, h_{B}, h_{R}\right)=\sqrt{\left(r \times 10^{3}\right)^{2}+\left(h_{B}-h_{R}\right)^{2}}, \\
& R_{\mathrm{bp}}=\left(10^{2 n_{h}} \frac{l_{\mathrm{bm}}(f, 1)}{l_{\mathrm{bm}}(f, 100)}\right)^{\frac{1}{\left(n_{h}-n_{l}\right)}}, \\
& n=\left\{\begin{array}{l}
0.1\left(24.9-6.55 \log h_{B}\right) \text { for } 1 k m \leq r \leq R_{\mathrm{bp}}, \\
2\left(3.27 \log h_{B}-0.67\left(\log h_{B}\right)^{2}-1.75\right) d, \text { for } R_{\mathrm{bp}} \leq r \leq 100 \mathrm{~km},
\end{array}\right.
\end{aligned}
$$

and $L_{\mathrm{bm}}$ is the basic median attenuation relative to free space, $r$ is the transmitter-receiver separation distance, $R_{\mathrm{bp}}$ is the breakpoint distance, $h_{B}$ and $h_{R}$ is the transmitter and receiver antenna height respectively, $v\left(h_{R}\right)$ is the receiver's reference height correction factor, $\mathrm{PL}_{\mathrm{fs}}$ is the free space path loss at distance $R, f$ represents the transmission frequency, $n_{h}$ and $n_{l}$ are the transmitter's effective height dependence of the higher and lower distance path loss exponent of the median attenuation relative to free space respectively, and $l_{\mathrm{bm}}$ is the frequency extrapolated basic median transmission relative to 
free space. For a suburban outdoor environment the eHata Pathloss model is,

$\mathrm{PL}_{\mathrm{eHs}}=\mathrm{PL}_{\mathrm{eH}}-\left(54.19-33.30 \log f+6.25(\log f)^{2}\right)$,

where $\mathrm{PL}_{\mathrm{eHs}}$ is the eHata Pathloss model for the suburban environment and $\mathrm{PL}_{\mathrm{eH}}$ is its urban equivalent presented in (12a)- (12f). Due to the large distance between the MNO interferers (eNodeB and UEs), and the ATC transceivers (ATC tower and the aircraft), we model the fading component as a log normal random variable to capture the effect of large scale fading.

\section{LiCENSEE System SPECTRUM EFFICIENCY}

We assume that perfect channel state information (CSI) is available at the transmitter. In cases where the incumbent system is not utilizing its spectrum, the licensee is able to transmit at maximum power to guarantee the desired signal to noise ratio (SNR) for each UEs according to its QoS requirement. In our model, the users are assumed to be randomly distributed according to (4) within the eNodB coverage area, thus the total system $\mathrm{SE}, \eta_{\mathrm{SE}}$, is the summation of the achievable bit rate for $K \mathrm{UEs}$,

$$
\eta_{\mathrm{SE}}=\sum_{k=1}^{K} \log _{2}\left(1+P_{k} g_{k}\right),
$$

where $g_{k}$ is the channel gain to noise ratio.

\section{A. Maximizing the Licensee Spectrum Efficiency}

If the LSA spectrum is unavailable, the licensee has to limit its transmit-power to ensure that the total interference power of the licensee (the MNO) at the incumbent receiver does not exceed the interference threshold. In other words, the transmit power should be reduced such that the incumbent's outage probability, $1-\mathcal{P}_{s}\{\theta\}$, does not exceed a given performance threshold, $\theta$, where $\left.\mathcal{P}_{s}\{\theta\}\right)=\operatorname{Pr}\{\operatorname{SINR}>\theta\}$ is the transmission success probability. Thus, while maximizing the achievable SE, the sum transmit power of the licensee must be such that the total interference caused to the incumbent does not cause outage.

To facilitate performance evaluation, and to differentiate uplink and downlink transmissions in our analysis we define $P_{U k}$ for the transmit power of the $k^{\text {th }}$ UE in the uplink, and equivalently $P_{B k}$ for the fraction of the eNodeB downlink transmitted power to the $k^{\text {th }}$ UE. Therefore, maximizing SE for the uplink is formulated as the following:

$$
\begin{aligned}
\eta_{\mathrm{SE}}^{*}= & \max _{P_{U}} \sum_{k=1}^{K} \log _{2}\left(1+P_{U k} g_{k}\right), \\
\text { s.t. } & \sum_{k=1}^{K} P_{U k} H_{k} l\left(r_{k}\right) \leq I_{\mathrm{th}},
\end{aligned}
$$

and for the downlink as:

$$
\eta_{\mathrm{SE}}^{*}=\max _{P_{B}} \sum_{k=1}^{K} \log _{2}\left(1+P_{B k} g_{k}\right),
$$

$$
\text { s.t. } P_{B} H_{B} l(r) \leq I_{\text {th }} \text {. }
$$

In (15) and (16), (15b) and (16b), are the constraint on the total interference from the licensee's transmissions in the uplink, and downlink, respectively, and $I_{t h}$ represents the incumbent's interference threshold, i.e., the maximum allowed interference for incumbents safe operation.

Since in the uplink, the interference constraint is imposed by multiple randomly distributed sources, to solve (15), the sum constraint on the interference power is decomposed as in [35], such that $I_{t h}=\sum_{k=1}^{K} I_{t h k}$. Thus (15) is rewritten as:

$$
\begin{aligned}
& \eta_{\mathrm{SE}}^{*}=\max _{P_{U}} \sum_{k=1}^{K} \log _{2}\left(1+P_{U k} g_{k}\right), \\
& \text { s.t. } \quad \sum_{k=1}^{K} P_{U} \cdot H_{k} \cdot \mathrm{PL}\left(v_{d k}, g_{d k}\right)-\sum_{k=1}^{K} I_{\mathrm{th} k} \leq 0, \\
& \quad P_{U k}>0 \quad\{k=1,2, \ldots \ldots, K\} .
\end{aligned}
$$

Using $P_{B}=\sum_{k=1}^{K} P_{B k},(16)$ is transformed to

$$
\eta_{\mathrm{SE}}^{*}=\max _{\left(P_{B}\right)} \sum_{k=1}^{K} \log _{2}\left(1+P_{B k} g_{k}\right),
$$

$$
\begin{aligned}
& \text { s.t. } \quad H_{B} \cdot \mathrm{PL}_{e H}\left(f, r, h_{B}, h_{R}\right) \cdot \sum_{k=1}^{K} P_{B k}-I_{\text {th }} \leq 0, \\
& \quad P_{B k}>0 \quad\{k=1,2, \ldots \ldots, K\} .
\end{aligned}
$$

In (17) and (18), (17c) and (18c) are the non-negative allocated power constraints for the uplink and downlink, respectively, and the corresponding optimization decision variables are $P_{U}=\left[P_{U 1}, \ldots, P_{U K}\right]$ and $P_{B}=\left[P_{B 1}, \ldots, P_{B K}\right]$. Furthermore, $\operatorname{PL}\left(v_{d k}, g_{d k}\right)$ is the path loss for the air -toground channel as a function of the height difference $v_{d k}$ and horizontal separation $g_{d k}$ between the $k^{\text {th }}$ UE and the aircraft while $\mathrm{PL}_{e H}\left(f, r, h_{B}, h_{R}\right)$ stands for the path loss between the eNodeB and the ATC tower as a function of the carrier frequency $f$, transmitter-receiver separation $r$ between them, $h_{B}$ is the eNodeB antenna height and $h_{R}$ is the ATC tower antenna height.

Using the Lagrangian method, we have:

$$
\begin{aligned}
& \mathcal{L}\left(P_{U k}, \lambda, v_{k}\right)= \\
& \sum_{k=1}^{K} \log _{2}\left(1+P_{U k} g_{k}\right) \\
&-\lambda\left(\sum_{k=1}^{K}\left(P_{U k} \cdot H_{k} \cdot \operatorname{PL}\left(v_{d k}, g_{d k}\right)-I_{t h k}\right)\right) \\
&+\sum_{k=1}^{K} v_{k} P_{U k},
\end{aligned}
$$


and for the downlink

$$
\begin{aligned}
\mathcal{L}\left(P_{\mathrm{B} k}, \lambda, v_{k}\right)= & \\
& \sum_{k=1}^{K} \log _{2}\left(1+P_{\mathrm{B} k} g_{k}\right) \\
& -\lambda\left(H_{B} \cdot \mathrm{PL}_{\mathrm{eH}}\left(f, r, h_{B}, h_{R}\right) \cdot \sum_{k=1}^{K} P_{\mathrm{B} k}-I_{\mathrm{th}}\right) \\
+ & \sum_{k=1}^{K} v_{k} P_{B k},
\end{aligned}
$$

where $\lambda \geq 0$ and $v_{k} \geq 0$ are Lagrangian multipliers for the interference and non negative power constraint. For the sake of brevity we will forthwith proceed with the solution of the uplink alone. Consequently, the Karush Kuhn Tucker (KKT) conditions [36] are:

$$
\begin{aligned}
\frac{\delta \mathcal{L}}{\delta P_{U k}}= & \frac{g_{k}}{\ln (2) \mathrm{PL}_{k} \cdot N_{k}\left(1+P_{U k} g_{k}\right)} \\
& -\lambda\left(H_{k} \mathrm{PL}\left(v_{d k}, g_{d k}\right)\right)+v_{k}=0
\end{aligned}
$$

$$
\lambda\left(\sum_{k=1}^{K} I_{\mathrm{th} k}-P_{U k} \cdot H_{k} \cdot \operatorname{PL}\left(v_{d k}, g_{d k}\right)\right)=0
$$

and

$$
\sum_{k=1}^{K} v_{k} P_{U k}=0
$$

for the stationarity condition (21a) and the complimentary slackness conditions (21b) \& (21c), respectively. If we assume that strict inequality holds in the non-negative power constraints of $(17 \mathrm{c})$, then by virtue of the complimentary slackness (21c), the Lagrange multiplier $v_{k}$ becomes zero. Thus in order to find the optimal allocated power $P_{k}^{*}$, we must also consider possible cases of having a non-positive power allocation in some channels.

In the first case, i.e., where $P_{k} \geq 0$ for all $k=1,2, \ldots, K$ : applying the KKT stationarity condition in (21a) we have,

$$
\begin{array}{r}
\frac{g_{k}}{\ln (2)}\left[\frac{\prod_{j \neq k}^{K}\left(1+P_{U j} g_{j}\right)}{1+P_{U k} g_{k} \prod_{j \neq k}^{K}\left(1+P_{U j} g_{j}\right)}\right] \\
=\lambda H_{k} \operatorname{PL}\left(v_{d k}, g_{d k}\right), \quad \forall k \in K,
\end{array}
$$

Therefore, the optimal allocated power $P_{U k}^{*}$ is

$$
P_{U k}^{*}=\left[\frac{1}{\lambda \ln (2)\left(H_{k} \mathrm{PL}\left(v_{d k}, g_{d k}\right)\right)}-\frac{1}{g_{k}}\right], \quad \forall k \in K
$$

Following similar steps, the solution to the optimization problems in (15)-(17), yields the equivalent optimal power allocation in the downlink transmission direction as

$$
\begin{array}{r}
P_{B k}^{*}=\left[\frac{1}{\lambda \ln (2)\left(H_{B} \mathrm{PL}_{e H}\left(f, r, h_{B}, h_{R}\right)\right)}-\frac{1}{g_{k}}\right], \\
\forall k \in K .
\end{array}
$$

In order to find the optimal allocated power $P_{k}^{*}$, in a situation where some channels have a non positive allocated power, we need to define and redistribute the available power to a set $K_{p} \subset K$ that contains strictly non-negative power allocations. In this case the optimal allocated power, $P_{\{U, B\} k}^{*}$, becomes

$$
\begin{aligned}
P_{U k}^{*}=\left[\frac{1}{\lambda \ln (2)\left(H_{k} \mathrm{PL}\left(v_{d k}, g_{d k}\right)\right)}-\frac{1}{g_{k}}\right], & \\
& \forall k \in K_{p} \mid P_{k}>0,
\end{aligned}
$$

and

$$
\begin{array}{r}
P_{B k}^{*}=\left[\frac{1}{\lambda \ln (2)\left(H_{B} \mathrm{PL}_{e H}\left(f, r, h_{B}, h_{R}\right)\right)}-\frac{1}{g_{k}}\right], \\
\forall k \in K_{p} \mid P_{k}>0 .
\end{array}
$$

Using (23) and (25) one can numerically determine the optimal $\lambda^{*}$ that gives $P_{U k}^{*}$ for the optimization problem in (15). Similarly, the optimal $\lambda^{*}$ that gives $P_{B k}^{*}$ for the optimization problem in (16) is obtained using (24) and (26).

\section{B. Optimal Power Allocation: Rated Transmit Power Con- straint}

In III-A, the formulated SE optimization problem only consider non-negative power allocation. In reality however, there is an upper bound imposed by the engineering specification on the allocated transmit power, which is the maximum transmit power rating of either the individual UE or the eNodeB. The rated transmit power is the manufacturer specified maximum power for each transmitting device. It is usually specified as effective isotropic radiated power (EIRP). Factoring this engineering design consideration into our SE maximisation, we can then re-formulate the optimization problem in (15) and (16) correspondingly. For the uplink transmission, the optimal power allocation for the $k^{\text {th }}$ user is upper bounded by the maximum transmit power rating. Thus, (15) simplifies to

$$
\begin{array}{cl}
\eta_{\mathrm{SE}}^{*}= & \max _{\left(P_{U}\right)} \sum_{k=1}^{K} \log _{2}\left(1+P_{U k} g_{k}\right), \\
\text { s.t. } \quad & \sum_{k=1}^{K} P_{U k} \cdot H_{k} \cdot \mathrm{PL}\left(v_{d k}, g_{d k}\right)-\sum_{k=1}^{K} I_{t h k} \leq 0, \\
& P_{U k}>0 \quad\{k=1,2, \ldots \ldots, K\}, \\
& P_{U k} \leq P_{\mathrm{Rk}} \quad\{k=1,2, \ldots \ldots, K\},
\end{array}
$$


where $P_{R k}$ is the rated power of each individual UE. In the uplink, the rated power constraint is for each individual transmitting node.

However, for the downlink case, the rated power constraint is a sum power constraint across all the receiving UEs. We thus re-formulate (16) as

$$
\begin{aligned}
& \eta_{\mathrm{SE}}^{*}=\max _{\left(P_{B}\right)} \sum_{k=1}^{K} \log _{2}\left(1+P_{B k} g_{k}\right), \\
& \text { s.t. } \quad H_{B} \cdot \mathrm{PL}_{e H}\left(f, r, h_{B}, h_{R}\right) \cdot \sum_{k=1}^{K} P_{B k}-I_{t h} \leq 0, \\
& \quad P_{B k}>0 \quad\{k=1,2, \ldots \ldots, K\}, \\
& \sum_{k=1}^{K} P_{B k} \leq P_{R B} \quad\{k=1,2, \ldots \ldots, K\},
\end{aligned}
$$

where $P_{R B}$ is the rated transmit power for the eNodeB. The constraint in (28d) is on the optimization decision variable $P_{B}$ itself and is strictly non binding since it can be directly implied by simply changing $P_{B}$ to $P_{R B}$ in the objective function.

\section{EFFiciency OF SPECTRUm UtILIZATION UNDER LSA}

The LSA spectrum utilization efficiency depends on the availability or unavailability of the spectrum. In this paper, we characterise the availability of the LSA spectrum within the incumbent's exclusion zone as a tandem queuing system with $Q$ multiple successive service layers. The $Q$ eNodeBs whose coverage area are located within the exclusion zone are characterised by $Q$ service layers. The arrival rate of the airplane landing or taking off at the airport is assumed to follow an exponential distribution. Therefore the LSA spectrum availability across all the $Q$ service layers is given as,

$$
\mathbf{X}=\left\{\mathbf{X}_{1}(j), \mathbf{X}_{2}(j), \ldots \mathbf{X}_{q}(j)\right\},
$$

where $\mathbf{X}_{q}(j)$ denotes the state space of $q$ th service layer and $j$ th service request (ATC communication with an aircraft).

We further describe $\mathbf{X}_{q}(\cdot)$ as a two state Markov chain analogous to a birth - death process. The first state (birth-to-death) describes the cases where the spectrum is being used by the ATC, while the second state (death-to-birth) characterizes the cases where the spectrum is available. For the sake of clarity we define the following parameters of the LSA spectrum availability tandem queueing system:

- $\gamma^{-1}$ : The time interval between successive flight take-off or landing(s), i.e., a cycle of the birth-death process,

- $\mu^{-1}$ : The duration of the ATC communication with an aircraft, i.e., duration of the spectrum occupancy(s) also referred to as busy period (BP),

- $\tau^{-1}$ : The duration of the spectrum vacancy(s), i.e., idle period (IP),

- $s$ : The LSA spectrum status given as $s \in\left\{s_{1}, s_{2}, \ldots s_{Q}\right\}$, $s_{q} \in\{0,1\}$, " 0 " where the spectrum is not available, and "1" where it is available,
- $j=\{0,1, \ldots, J\}$ : The number of aircraft landings or take-offs in the airport (service request) at different times with an exponential arriving rate, $\gamma \in\left\{\gamma_{1}, \gamma_{2} \ldots \gamma_{Q}\right\}$, and service rate, $\mu \in\left\{\mu_{1}, \mu_{2}, \ldots \mu_{Q}\right\}$.

Based on the above, LSA spectrum utilization for each service layer (eNodeB coverage area) is described by the state space equation,

$$
\mathbf{X}_{q}=\{(j, s), \in\{0,1, \ldots, J\} \times\{0,1\}\} .
$$

Similarly, $\tau^{-1} \in\left\{\tau_{1}^{-1}, \tau_{2}^{-1}, \ldots \tau_{q}^{-1}\right\}$, and $\operatorname{Pr}\{$ ATC $\} \in$ $\{\operatorname{Pr}\{$ ATC 1$\}, \operatorname{Pr}\{$ ATC 2$\}, \ldots, \operatorname{Pr}\{$ ATCq $\}\}$, where $\operatorname{Pr}\{$ ATC $\}$ is the probability of ATC transmission occurring during the time interval between successive flight take-offs or landings, i.e., the probability of the LSA spectrum being busy, the distribution of which is given by Laplace-Stieltjes transform [37]:

$$
\mathcal{L}_{P_{\text {ATC }}}(s)=\frac{1}{2 \gamma}\left(\gamma+\mu+s-\sqrt{(\gamma+\mu+s)^{2}-4 \gamma \mu}\right) .
$$

Thus three following scenarios can be deduced from the process described above:

- An aircraft landing/taking-off service request is being handled and there is telemetry communication with an aircraft within and around the coverage area of a particular eNodeB. Thus the spectrum is busy or unavailable,

- There is still an ongoing ATC communication with an aircraft, but the aircraft is not within the coverage area of the particular eNodeB, hence the spectrum is free or available for unrestricted licensee communication,

- There is no ATC transmission hence the spectrum is free or available across all $q$ service layers.

Spectrum utilization efficiency is usually measured in time and space dimension. However, we define the LSA spectrum utilization efficiency, $\eta_{U T} \in\left\{\eta_{U T 1}, \eta_{U T 2}, \ldots, \eta_{U T Q}\right\}$ as a utility function of the $v_{q}$, the effective server's (in this case the LSA spectrum) busy period ratio of each layer to all the service layers, and the achievable $\mathrm{SE} \eta_{\mathrm{SE} q}$ for each $q$ successive service layer (eNodeB coverage area) where the spectrum is not available or occupied by the incumbent.

$$
\mathrm{U}_{v_{q}}\left(\eta_{\mathrm{SE} q}\right)=\left\{\begin{array}{lc}
\left(1-v_{q}\right) \mathrm{SE} q_{\max }+v_{q} \cdot \eta_{\mathrm{SE} q}, & 0<v_{q}<1 \\
\mathrm{SE} q_{\max }, & v_{q}=0 \\
\eta_{\mathrm{SE} q}, & v_{q}=1
\end{array}\right.
$$

where $v_{q}$ is given by $\frac{\mu}{\mu_{q}}$ for $q=\{1,2, \ldots, Q\}$. SE $q_{\text {max }}$ is the maximum achievable system SE when the licensee transmission is not constrained by the incumbent's operational activities, i.e., where the spectrum is free.

The first part of (32) occurs when the incumbent and the licensee transmission shadow radius intersects. In the uplink direction, when this occurs, the spectrum utilization efficiency becomes a utility measure of the ratio of each eNodeB BP to the total duration of the service time. For this to occur, the distance between the aircraft and the UEs must be greater than the summation of the transmission shadow of the aircraft and the UEs. Since the eNodeB and the ATC tower is stationary, this scenario does not apply in the downlink direction.

However the second and third equation in the utility function, in (32), defines the utilization efficiency for distant and 
TABLE III: Simulation Parameters

\begin{tabular}{ll}
\hline Parameter & Value \\
\hline eNodeB Radius & $100,250,500,1000$ (metres) \\
No. of UE & $5,10,25,100$ \\
Downlink Transmit Power & $0.2-15.85 \mathrm{w}(23-42 \mathrm{dBm})$ \\
Uplink Transmit Power & $0.2-2.52 \mathrm{w}(23-34 \mathrm{dBm})$ \\
Noise Spectral Density & $-60 \mathrm{dBm} / \mathrm{Hz}$ \\
eNodeB Antenna Height & 30 metres \\
UE Antenna Height & 1.5 metres \\
ATC Type-B Receiver Noise Figure(NF) & $3 \mathrm{~dB}$ \\
Boltzmann's constant(k) & $1.38 \times 10^{-23}$ \\
Temperature (T) & $290 \mathrm{Kelvin}$ \\
Noise Power & $10 l o g(\mathrm{kTB})+\mathrm{NF}$ \\
Protection Ratio (I/N) & $-10 \mathrm{~dB}$ \\
Bandwidth (B) & $10 \mathrm{MHz}$ \\
LSA Frequency Band & $2300-2400 \mathrm{MHz}$ \\
Career Frequency & $2350 \mathrm{MHz}$ \\
Height of ATC Tower & $8 \mathrm{metres}$ \\
Airplane take-off angle & $7-25 \mathrm{degrees}$ \\
Airplane take-off speed & $65 \mathrm{~m} / \mathrm{s}$ \\
Airplane Acceleration & $0.29 \mathrm{~m} / \mathrm{s}^{2}$ \\
\hline
\end{tabular}

close eNodeB coverage areas respectively. In the former case, at a certain distance, the interference generated by the eNodeB is significantly less than the the interference threshold of the ATC system, hence the MNO licensee can operate at its rated transmit power. In the latter, for eNodeB coverage areas close to the ATC tower, the MNO must adjust its transmission power to prevent harmful interference to the incumbent's system, hence the maximum achievable rate for the total duration of the ATC communication is the constrained busy spectrum SE $\eta_{S E q}$, for those eNodeB coverage areas. Furthermore, the second equation of (32) also applies to the uplink SE in those distant eNodeB coverage areas where the aircraft has attained considerable height such that the distance separation between it and UEs on the ground is more than their shadow radius combined.

\section{Simulation Results And Analysis}

The simulation parameters are summarised in Table III. We simulate a circular geographical area with a radius of 200 $\mathrm{km}$ centred at the airport consisting of several eNodeBs. The closest eNodeB to the ATC tower is further than $1 \mathrm{~km}$. The UEs are assumed to be distributed in the cell area according to (4). The ascent or glide angle (take-off angle) is assumed to change at the rate of 1 degrees per second while the cruising speed is taken as $244.44 \mathrm{~m} / \mathrm{s}$ (475.16 knots). The ATG propagation parameters used are for the urban environment. Furthermore, we assume co-channel interference between both systems, the eNodeB antenna gain is set to $17 \mathrm{~dB}$, the feeder loss is taken as $3 \mathrm{~dB}$, the telemetry receiver main lobe antenna gain is equal to $45 \mathrm{dBi}$, and $1 \mathrm{~dB}$ is its feeder loss as specified in [38].

We first investigate the performance of the licensee system SE optimization using the optimal power allocation in the downlink and then make a comparative analysis with the uplink. In Fig. 2 the SE is given versus the transmit power for
10 and 5 UEs in the downlink transmission for both the system with our proposed optimal power allocation and without. As it is seen, there is a significant improvement in the system SE with the optimal power allocation proposed. Judging by the graph for 10 UEs, around seven fold (700\%) improvement is obtained over the system without the optimal power allocation.

In Fig. 3 we show the SE gain for different number of UEs versus transmit power. It is seen that the achieved SE gain is directly proportional to the number of UEs similar to Fig. 2 , where the plot for the larger number of UEs is expectedly higher than the one for smaller number of UEs. This means that the SE gain increases proportionately with increasing number of UEs. Furthermore, to show the actual increase in the SE, we introduce a comparative metric, a decibel SE gain. Interestingly this revealed further facts not only about the SE gain pattern in relation to the number of UEs, but also with increasing the operating transmit power.

In Fig. 3 the decibel SE gain also indicates that a larger SE improvement is obtained at lower transmit power. Moreover, in comparison to the linear SE gain in $\mathrm{b} / \mathrm{s} / \mathrm{Hz}$, the decibel SE gain shows an approximately equal value at low transmit power for users 10, 25 and 100 at low transmit power while the graph becomes more distinct with increasing operating power. In contrast to the linear SE gain, the decibel SE gain has an inverse proportion to the number of UEs in the system. In the plot for the SE gain in $\mathrm{b} / \mathrm{s} / \mathrm{Hz}$, higher number of UEs has a higher actual SE gain value than normal, however the decibel SE gain showed that lower number of UEs recorded a better gain ratio than higher number of UEs. This can be explained by the fact that at lower number of UEs, the interference to the incumbent system is low, thus the transmit power reduction required is relatively small and there is a higher degree of freedom to take advantage of the optimal power allocation.

In Fig. 4, we investigate the effect of different cell sizes on the decibel SE gain. Similar trend is seen for various sizes of

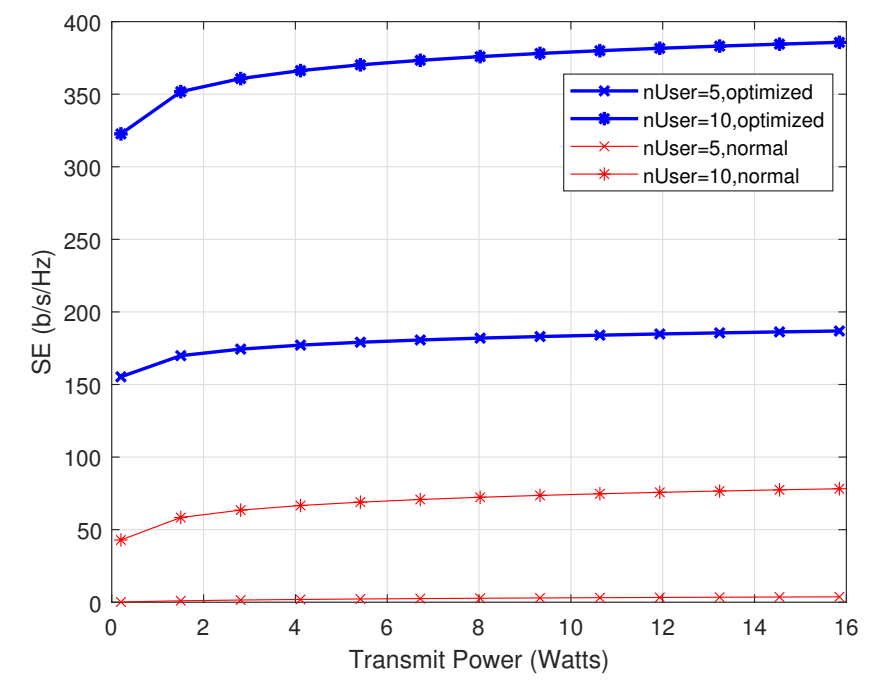

Fig. 2: Comparison of the SE in optimized and the nonoptimized systems $v s$. total transmit power. 

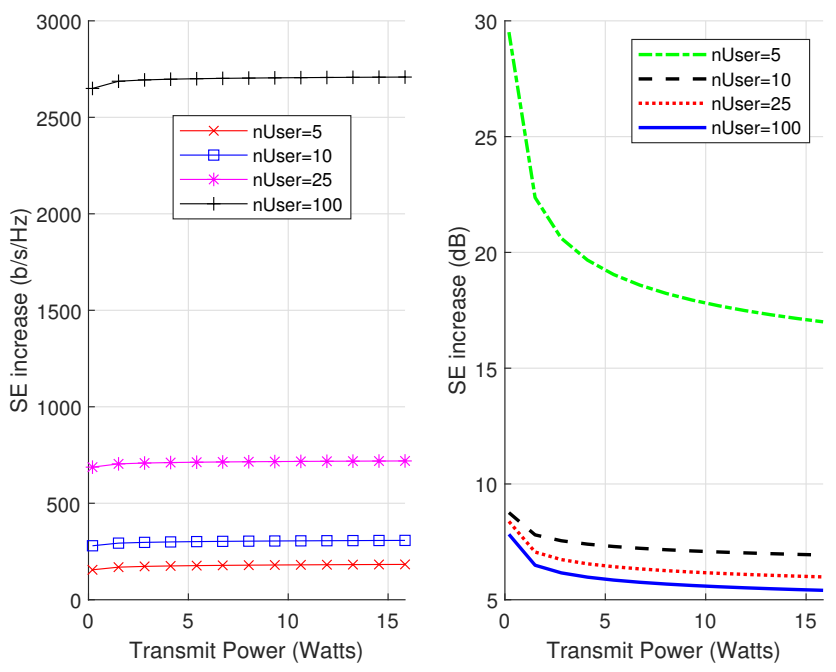

Fig. 3: Downlink SE gain vs. transmit power.

eNodeB radius as in the second graph of Fig. 3. We further notice that the SE gain, increases with increasing eNodeB coverage radius. Similar trend is also seen in the plot for the 5 UEs and 10 UEs, where the gap shows a slight increase with increasing eNodeB coverage radius. Similar trend is observed for the uplink.

Fig. 5 shows the plot of the decibel SE gain vs. transmit power in the uplink transmission direction. Similar to the downlink decibel SE gain, the uplink SE gain is inversely proportional to the number of UEs. However, there is a difference in the shape of the curve. While for the downlink, the decibel SE gain is a monotonically decreasing curve, in the uplink the decibel SE gain curve initially increases to a peak value after which it gradually decreases. The implication of this is that at very low transmit power the advantage provided by the optimal power allocation is small. By increasing the transmit power, the effect of the optimal power allocation becomes more significant, after which it starts to decrease.

\section{A. Efficiency of the LSA Spectrum Utilization}

For ease of analysis, we focus on the eNodeB radius of $1000 \mathrm{~m}$, hence for a distance of $200 \mathrm{~km}$ from the airport, we have a total of hundred (100) $q$ service layers in our utilization efficiency analysis. The busy period ratio $v_{q}$ for each service layer to all service layers is obtained using the procedure in Algorithm 1.

Fig. 6 shows the interference power from the eNodeB to the ATC tower for different eNodeB coverage areas within the considered geographical radius. To better visualize, we plotted the $y$-axis as a log scale in the second graph of Fig. 6. In the first graph because of the margin of difference between the interference generated by the first eNodeB and the second one, it was practically impossible to make any comparison even for just the first two eNodeB coverage areas. In the second graph, it was possible to plot the interference of all the eNodeB coverage areas and compare them. It is seen that for a high eNodeB transmit power, the interference power generated by

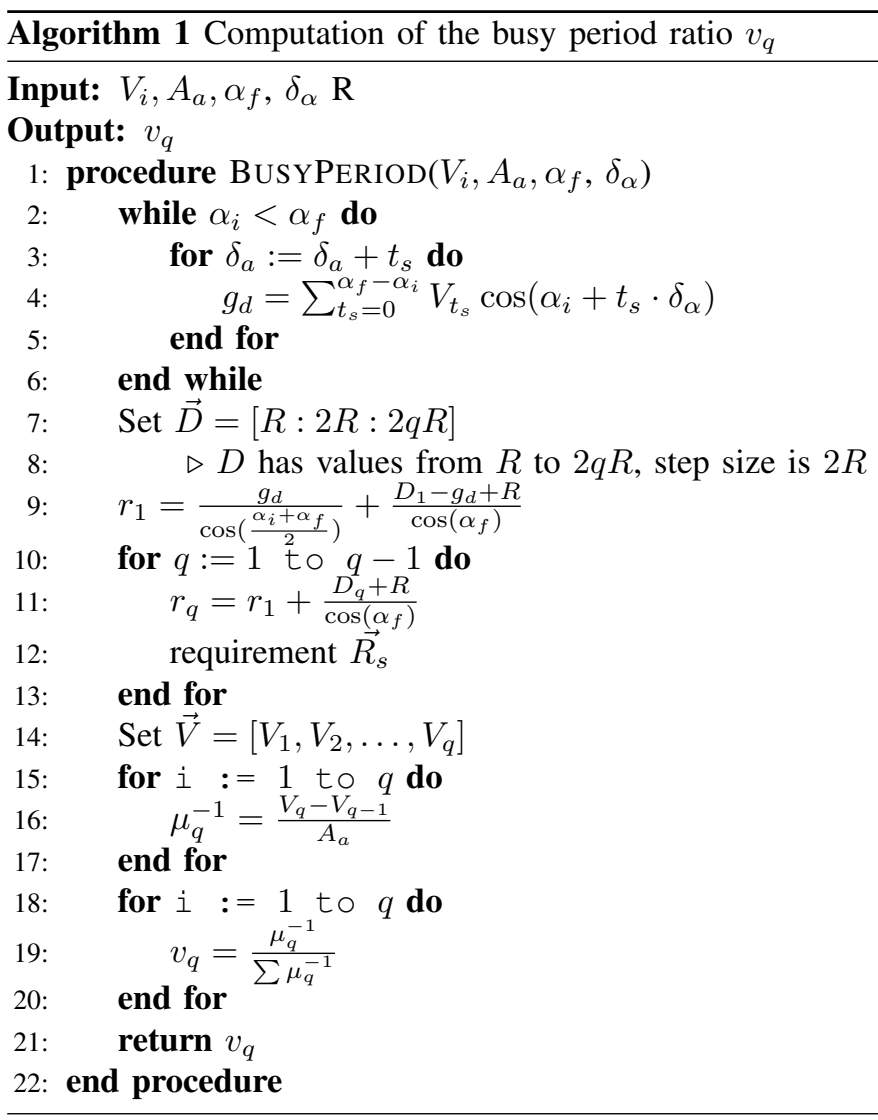

the licensee is still higher than the incumbent threshold even at the 100th eNodeB, which is about $200 \mathrm{~km}$ away from the ATC tower. This is in agreement with the report of the compatibility studies done by the electronic communications committee in [38], which gives separation distance between an MNO and ATC to be in order of hundreds of kilometres. However, at a low transmit power, starting from the 50th eNodeB (about 100 $\mathrm{km}$ distance from the ATC tower), the received interference by the ATC tower is below the prescribed threshold.

The implication of the above observations of Fig. 6 from the utility function of (32) is that the spectrum utilization efficiency at higher transmit powers and coverage areas close to the airport reduces to $\eta_{S E}$ since the eNodeBs have to maintain their power reduction policy for the total duration of the ATC tower communication with an airplane while it is still within its airspace. However, for further eNodeBs, as well as low transmit power below a certain threshold even from about $100 \mathrm{~km}$ distance from the ATC tower, the licensee can operate at its rated transmit power hence the spectrum utilization efficiency is given by the second part of (32).

The bar chart in Fig. 7 shows the BP ratio $v_{q}$ across different eNodeBs. As it is seen, $v_{q}$ decreases with increasing separation distance between the eNodeB coverage area and the airport. This is because of the increase in the airplane speed as it accelerates across the area. The implication of this on the spectrum utilization efficiency is that as a certain eNodeB coverage becomes further removed from the vicinity of the airport, the time for operating under the power reduction policy becomes reduced. To put this in a better context we analyse 

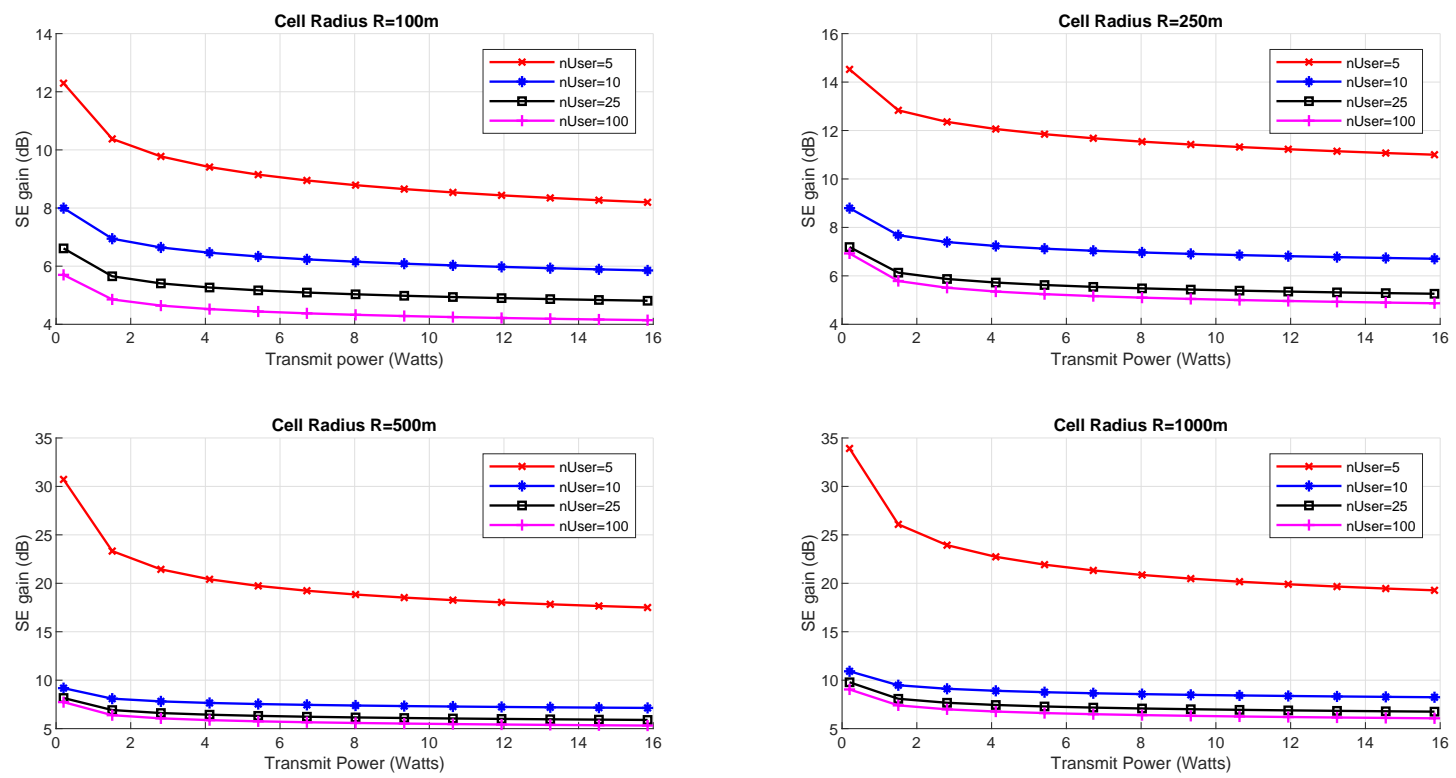

Fig. 4: Downlink SE gain vs. transmit power for various eNodeB radius and number of users.

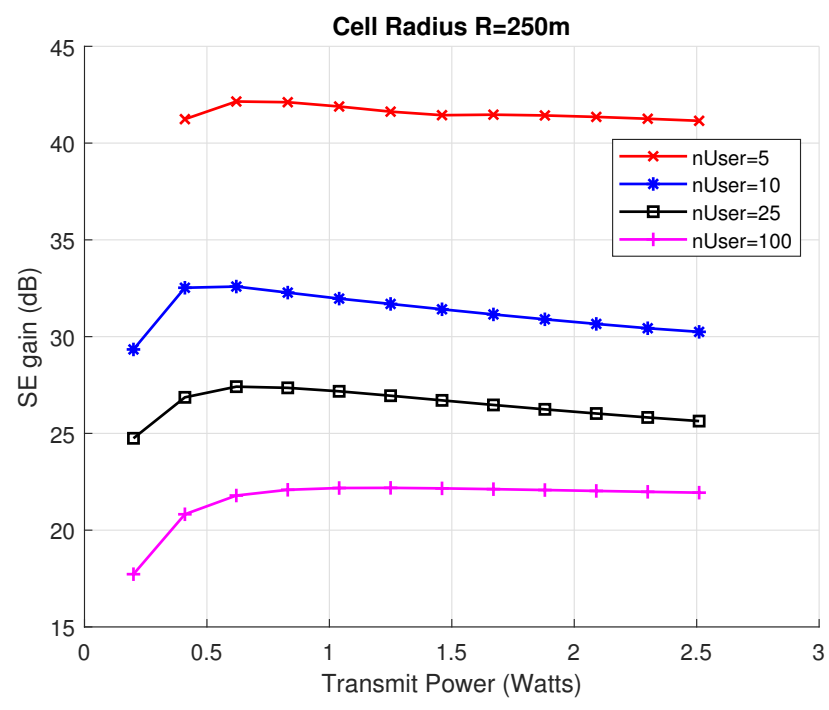

Fig. 5: Uplink SE gain $v s$. transmit power for various number of users, where $R=250 \mathrm{~m}$.

the first equation in the utility function of (32). From the first equation of the utility function of (32), it is seen that better spectrum utilization is obtained when the first part of the equation is high, i.e., when the licensee can operate at its maximum transmit power. A high value of $v_{q}$ reduces the time the licensee can operate at full power and increases the length of the period it operates under reduced power policy, thus effectively reducing the utilization efficiency.

The above is further confirmed by the graphs in Fig. 8. It is seen from the first graph, (Fig. 8a), that the achievable SE in the uplink for eNodeB coverage areas farther from the airport is higher than those closer. Thus, for those distant coverage
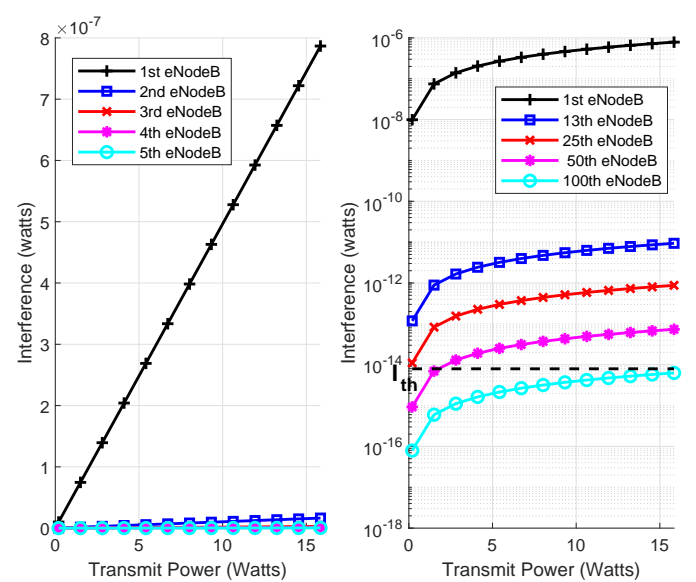

Fig. 6: Downlink Interference power for different eNodeB.

areas, the spectrum utilization efficiency is improved not only because of the smaller $v_{q}$ which minimizes the period for limited power regime but also by higher achievable $\eta_{\mathrm{SE}}$. The monotonically decreasing graphs in Fig. 8c, suggests that the achievable SE has an inverse relationship with the busy period ratio of each service layer to all service layers.

Fig. 8b shows the uplink interference power at the airplane for selected transmit power levels across the coverage areas within the considered radius around the airport. Similar to the downlink, the interference reduces for eNodeB coverage area farther away from the airport. However this is not due to the distance to the airport but rather due to the increasing height between the airplane and the licensee UEs. Unlike the downlink case, the airplane could receive interference significantly higher than the prescribed threshold at the farthest eNodeB coverage area even at the lowest transmitting power. 


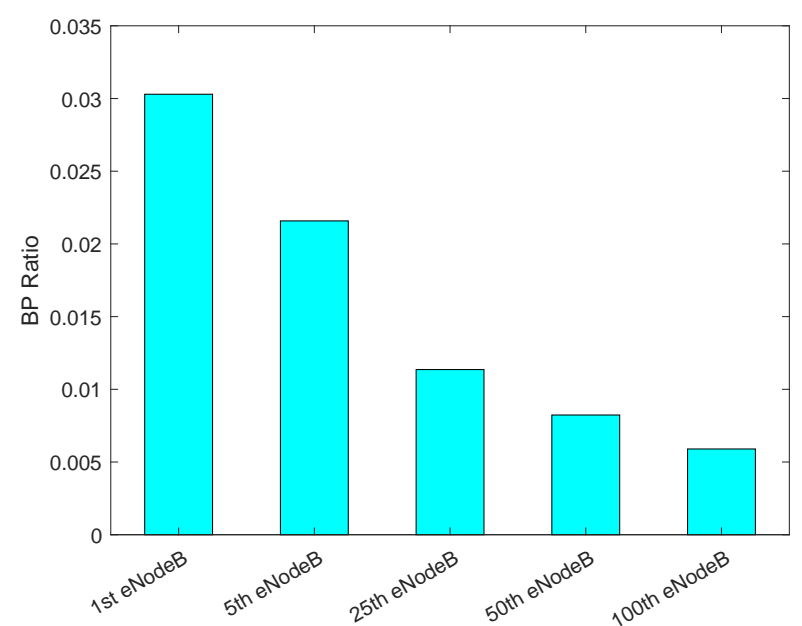

Fig. 7: BP ratio, $v_{q}$, for different eNodeB.

This can be attributed to the better LoS in the ATG propagation path between the UEs and the airplane. As a result of higher probability of LoS, the signal attenuation is smaller compared to the terrestrial path loss model in the downlink.

This is confirmed by the interference power from the UE to the ATC tower shown in Fig. 9, which has a terrestrial propagation similar to the downlink. For the same transmit power and separation distance, it is seen in Fig. 9 that the interference power is several orders of magnitude lower than it was in Fig. 8b. At low transmit power the obtained results, show that the received interference at the ATC tower from the UE is below the prescribed threshold at approximately 23 $\mathrm{km}$ distance, i.e., the 12th eNodeB. This suggests that instead of suspending licensee transmission in all the 100 eNodeBs and even farther as dictated by the exclusion zone policy [39], [40], the licensee can operate under the full transmit power in the uplink starting from the 13th eNodeB.

\section{CONCLUSION}

In this paper, we investigated a LSA sharing arrangement between an ATC incumbent and a MNO licensee, during the period when the incumbent is utilizing its spectrum for telemetry services. We consider a circular protection radius of $200 \mathrm{~km}$ with many eNodeBs located within this geographical radius. We then optimize the licensee system SE while ensuring the incumbent's interference threshold is not exceeded. In addition, we proposed a utility function of achievable SE and busy period ratio of each service layer to all service layers, as a metric for measuring the additional spectrum utilization efficiency during the period of the incumbents occupation of its spectrum. Results show that the SE is improved by at least seven times (700\%) with the proposed optimal power allocation. Furthermore, the introduced decibel SE gain measure reveals that the UE traffic in a eNodeB coverage area is inversely proportional to the achieved SE improvement obtained when using the proposed optimal power allocation.

The implication of this is that for practical LSA deployment scenario, optimal system design must be geared towards achieving the best trade-off between the UE traffic and the desired SE. Moreover, considering the possibility of the LSA system co-existing with the legacy MNO network, this result provides a guide for reliable and optimal traffic distribution between the two systems. It is also seen that the farther the eNodeB coverage area is to the airport, the better is the achievable SE. This is due to reduction in interference power from the licensee to the ATC system. In practical terms, the implication of this is that at farther distance from the airport, the operating parameters of the MNO in an LSA system can be configured with less stringent restrictions. Due to the higher LoS in the ATG path between the licensee interferer and the incumbent airborne receiver, it is seen that the interference suffered by the uplink of the incumbent system persists to far greater distance than received interference in its downlink. In fact, the interfering signal from the licensee UEs to the incumbent downlink receiver (the ATC tower), drops within the tolerated threshold at a considerably shorter distance (when compared to the equivalent uplink scenario).

\section{REFERENCES}

[1] Cisco, "Cisco Visual Networking Index: Forecast and Methodology, 2016 2021," "White Paper", Tech. Rep., June, 2017.

[2] E. Markova, I. Gudkova, A. Ometov, I. Dzantiev, S. Andreev, Y. Koucheryavy, and K. Samouylov, "Flexible Spectrum Management in a Smart City Within Licensed Shared Access Framework," IEEE Access, vol. 5, pp. 22 252-22 261, 2017.

[3] K. Laehetkangas, H. Saarnisaari, and A. Hulkkonen, "Licensed Shared Access System Development for Public Safety," in European Wireless 2016; 22nd European Wireless Conference, May 2016, pp. 1-6.

[4] P. Masek, E. Mokrov, A. Pyattaev, K. Zeman, A. PonomarenkoTimofeev, A. Samuylov, E. Sopin, J. Hosek, I. A. Gudkova, S. Andreev, V. Novotny, Y. Koucheryavy, and K. Samouylov, "Experimental Evaluation of Dynamic Licensed Shared Access Operation in Live 3GPP LTE System," in 2016 IEEE Global Communications Conference (GLOBECOM), Dec 2016, pp. 1-6.

[5] M. Palola, M. Matinmikko, J. Prokkola, M. Mustonen, M. Heikkil, T. Kippola, S. Yrjl, V. Hartikainen, L. Tudose, A. Kivinen, J. Paavola, and K. Heiska, "Live Field Trial of Licensed Shared Access (LSA) Concept using LTE Network in $2.3 \mathrm{GHz}$ Band," in 2014 IEEE International Symposium on Dynamic Spectrum Access Networks (DYSPAN), April 2014, pp. 38-47.

[6] M. Palola, M. Matinmikko, J. Prokkola, M. Mustonen, M. Heikkil, T. Kippola, S. Yrjl, V. Hartikainen, L. Tudose, A. Kivinen, J. Paavola, K. Heiska, T. Hnninen, and J. Okkonen, "Description of Finnish Licensed Shared Access (LSA) field trial using TD-LTE in $2.3 \mathrm{GHz}$ band," in 2014 IEEE International Symposium on Dynamic Spectrum Access Networks (DYSPAN), April 2014, pp. 374-375.

[7] M. Palola, T. Rautio, M. Matinmikko, J. Prokkola, M. Mustonen, M. Heikkil, T. Kippola, S. Yrjl, V. Hartikainen, L. Tudose, A. Kivinen, J. Paavola, J. Okkonen, M. Mkelinen, T. Hnninen, and H. Kokkinen, "Licensed Shared Access (LSA) Trial Demonstration using Real LTE Network," in 2014 9th International Conference on Cognitive Radio Oriented Wireless Networks and Communications (CROWNCOM), June 2014, pp. 498-502.

[8] M. Matinmikko, M. Palola, M. Mustonen, T. Rautio, M. Heikkil, T. Kippola, S. Yrjl, V. Hartikainen, L. Tudose, A. Kivinen, H. Kokkinen, and M. Mkelinen, "Field Trial of Licensed Shared Access (LSA) with Enhanced LTE Resource Optimization and Incumbent Protection," in 2015 IEEE International Symposium on Dynamic Spectrum Access Networks (DySPAN), Sept 2015, pp. 263-264.

[9] J. Kalliovaara, T. Jokela, R. Ekman, J. Hallio, M. Jakobsson, T. Kippola, and M. Matinmikko, "Interference Measurements for Licensed Shared Access (LSA) Between LTE and Wireless cameras in $2.3 \mathrm{GHz}$ band," in 2015 IEEE International Symposium on Dynamic Spectrum Access Networks (DySPAN), Sept 2015, pp. 123-129.

[10] D. Guiducci, C. Carciofi, V. Petrini, S. Pompei, J. Llorente, V. Ferrer, J. Costa-Requena, E. Spina, G. D. Sipio, D. Massimi, D. Spoto, F. Amerighi, T. Magliocca, H. Kokkinen, P. Chawdhry, L. Ardito, 


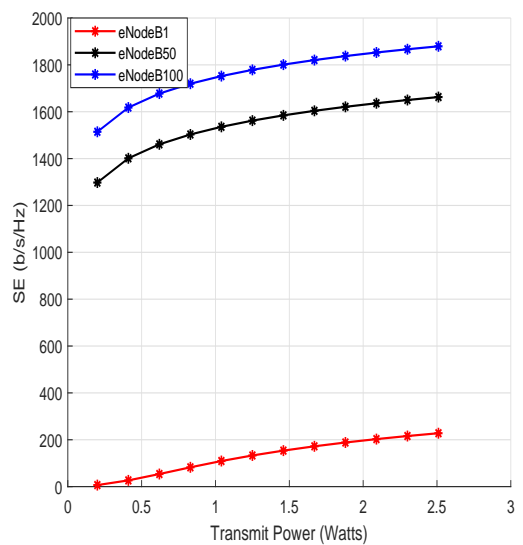

(a) Uplink SE gain for different eNodeB.

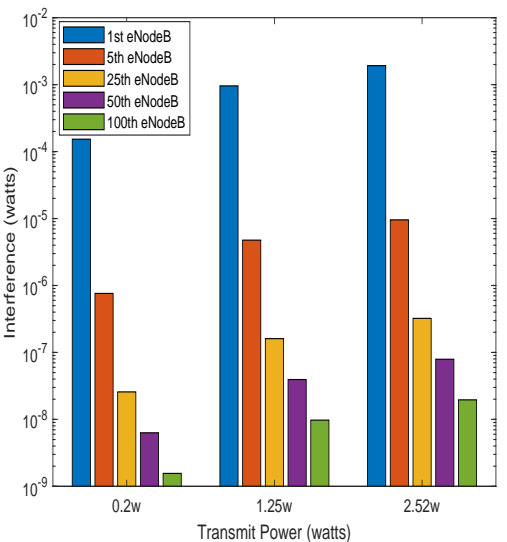

(b) Uplink Interference power for different eNodeB.

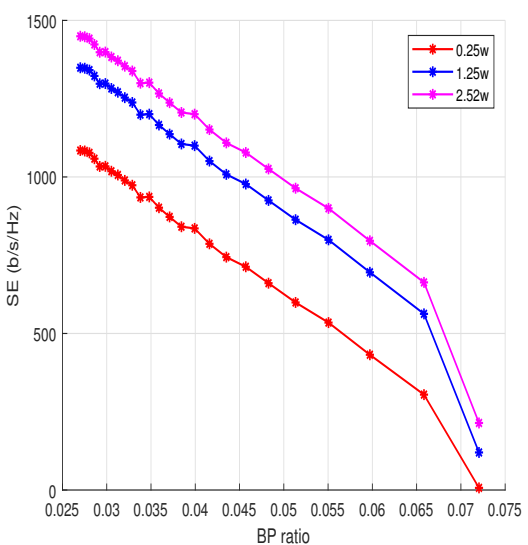

(c) SE vs. BP ratio $v_{q}$, for different eNodeB.

Fig. 8: Uplink SE, interference power and $v_{q}$ for eNodeBs across different separation distance.

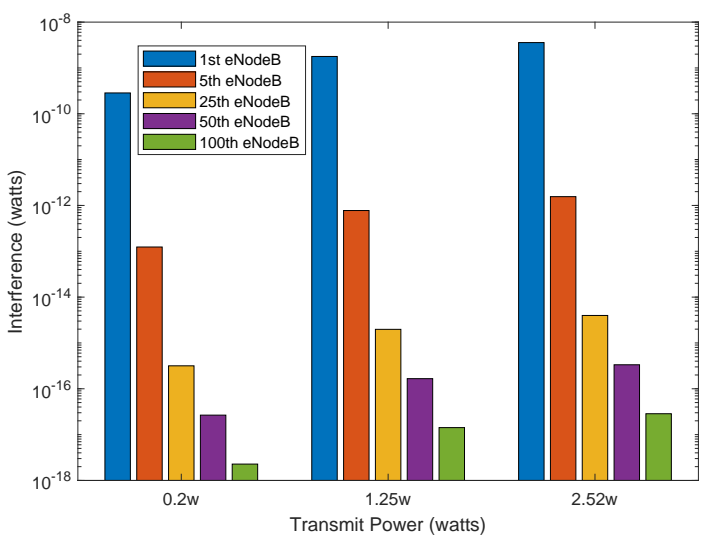

Fig. 9: Uplink interference to ATC tower.

S. Yrjola, V. Hartikainen, L. Tudose, P. Muller, M. Gianesin, F. Grazioli, and D. Caggiati, "Sharing Under Licensed Shared Access in a Live LTE Network in the 2.32.4 GHz Band End-to-End Architecture and Compliance Results," in 2017 IEEE International Symposium on Dynamic Spectrum Access Networks (DySPAN), March 2017, pp. 1-10.

[11] "ECC Report 205: Licensed Shared Access (LSA), CEPT Working Group Frequency Management," February 2014

[12] Deloitte, “"'The Impacts of Licensed Shared Use-of-Spectrum, report for GSM Association," January 2014.

[13] A. Ponomarenko-Timofeev, A. Pyattaev, S. Andreev, Y. Koucheryavy, M. Mueck, and I. Karls, "Highly Dynamic Spectrum Management within Licensed Shared Access Regulatory Framework," IEEE Communications Magazine, vol. 54, no. 3, pp. 100-109, March 2016.

[14] "Final RSPG Report on Collective Use of Spectrum and Other Sharing Approaches," November 2011.

[15] E. Mokrov, A. Ponomarenko-Timofeev, I. Gudkova, P. Masek, J. Hosek, S. Andreev, Y. Koucheryavy, and Y. Gaidamaka, "Transmit Power Reduction for a Typical Cell With Licensed Shared Access Capabilities," IEEE Transactions on Vehicular Technology, vol. 67, no. 6, pp. 55055509, June 2018.

[16] Fp7 project adel. [Online]. Available: http://www.fp7-adel.eu

[17] V. Frascolla, A. J. Morgado, A. Gomes, M. M. Butt, N. Marchetti, K. Voulgaris, and C. B. Papadias, "Dynamic Licensed Shared Access - A New Architecture and Spectrum Allocation Techniques," in 2016 IEEE 84th Vehicular Technology Conference (VTC-Fall), Sept 2016, pp. $1-5$.

[18] I. Gudkova, E. Markova, P. Masek, S. Andreev, J. Hosek, N. Yarkina, K. Samouylov, and Y. Koucheryavy, "Modeling the Utilization of a
Multi-Tenant Band in 3GPP LTE System with Licensed Shared Access," in 2016 8th International Congress on Ultra Modern Telecommunications and Control Systems and Workshops (ICUMT), Oct 2016, pp. 119123.

[19] B. A. Jayawickrama, E. Dutkiewicz, and M. Mueck, "Incumbent User Active Area Detection for Licensed Shared Access," in 2015 IEEE 82nd Vehicular Technology Conference (VTC2015-Fall), Sep. 2015, pp. 1-5.

[20] I. Gudkova, K. Samouylov, D. Ostrikova, E. Mokrov, A. PonomarenkoTimofeev, S. Andreev, and Y. Koucheryavy, "Service Failure and Interruption Probability Analysis for Licensed Shared Access Regulatory Framework," in 2015 7th International Congress on Ultra Modern Telecommunications and Control Systems and Workshops (ICUMT), Oct 2015, pp. 123-131.

[21] V. Y. Borodakiy, K. E. Samouylov, I. A. Gudkova, D. Y. Ostrikova, A. A. Ponomarenko-Timofeev, A. M. Turlikov, and S. D. Andreev, "Modeling unreliable lsa operation in 3gpp lte cellular networks," in 2014 6th International Congress on Ultra Modern Telecommunications and Control Systems and Workshops (ICUMT), Oct 2014, pp. 390-396.

[22] I. Gudkova, A. Korotysheva, A. Zeifman, G. Shilova, V. Korolev, S. Shorgin, and R. Razumchik, "Modeling and Analyzing Licensed Shared Access Operation for 5G Network as an Inhomogeneous Queue with Catastrophes," in 2016 8th International Congress on Ultra Modern Telecommunications and Control Systems and Workshops (ICUMT), Oct 2016, pp. 282-287.

[23] K. Ntougias, C. B. Papadias, G. K. Papageorgiou, and G. Hasslinger, "Spectral Coexistence of 5G Networks and Satellite Communication Systems Enabled by Coordinated Caching and QoS-Aware Resource Allocation," in 2019 27th European Signal Processing Conference (EUSIPCO), 2019, pp. 1-5.

[24] G. K. Papageorgiou, K. Voulgaris, K. Ntougias, D. K. Ntaikos, M. M. Butt, C. Galiotto, N. Marchetti, V. Frascolla, H. Annouar, A. Gomes, A. J. Morgado, M. Pesavento, T. Ratnarajah, K. Gopala, F. Kaltenberger, D. T. M. Slock, F. A. Khan, and C. B. Papadias, "dvanced Dynamic Spectrum 5G Mobile Networks Employing Licensed Shared Access," IEEE Communications Magazine, vol. 58, no. 7, pp. 21-27, 2020.

[25] C. Galiotto, G. K. Papageorgiou, K. Voulgaris, M. M. Butt, N. Marchetti, and C. B. Papadias, "Unlocking the Deployment of Spectrum Sharing With a Policy Enforcement Framework," IEEE Access, vol. 6, pp. 11 793-11 803, 2018.

[26] E. Prez, K. Friederichs, I. Viering, and J. Diego Naranjo, "Optimization of Authorised/Licensed Shared Access resources," in 2014 9th International Conference on Cognitive Radio Oriented Wireless Networks and Communications (CROWNCOM), June 2014, pp. 241-246.

[27] N. Taramas, G. C. Alexandropoulos, and C. B. Papadias, "Opportunistic Beamforming for Secondary Users in Licensed Shared Access Networks," in 2014 6th International Symposium on Communications, Control and Signal Processing (ISCCSP), May 2014, pp. 526-529.

[28] S. Onidare, K. Navaie, and Q. Ni, "Maximum Achievable Sum Rate in Highly Dynamic Licensed Shared Access," in 2019 IEEE 89th Vehicular Technology Conference (VTC2019-Spring), April 2019, pp. 1-5. 
[29] M. Haenggi, Stochastic Geometry for Wireless Networks, 1st ed. New York, NY, USA: Cambridge University Press, 2012.

[30] A. Al-Hourani, S. Kandeepan, and S. Lardner, "Optimal LAP Altitude for Maximum Coverage," IEEE Wireless Communications Letters, vol. 3 , no. 6, pp. 569-572, Dec 2014.

[31] S. R. Saunders and A. Argo-Zavala, "Antennas and Propagation for Wireless Communication Systems, 2nd Edition,” New York:Wiley, 2007.

[32] J. Holis and P. Pechac, "Elevation Dependent Shadowing Model for Mobile Communications via High Altitude Platforms in Built-Up Areas," IEEE Transactions on Antennas and Propagation, vol. 56, no. 4, pp. 1078-1084, April 2008.

[33] ITU-R, "Propagation Data and Prediction Methods Required for the Design of Terrestrial Broadband Millimetric Radio Access Systems Operating in a Frequency Range about 20 to $50 \mathrm{GHz}$," Recommendation P.1410-2, 2005, ITU.

[34] E. Drocella, J. Richards, R. Sole, F. Najmy, A. Lundy, and P. McKenna, "3.5-GHz-Exclusion Zone Analyses and Methodology, NTIA report 15517," March 2016.

[35] Y. Wei, "Sum-Capacity Computation for the Gaussian Vector Broadcast Channel via Dual Decomposition," IEEE Transactions on Information Theory, vol. 52, no. 2, pp. 754-759, Feb 2006.

[36] S. Boyd and L. Vandenberghe, Convex Optimization. New York, NY, USA: Cambridge University Press, 2004.

[37] I. Adan and J. Resing, Queueing Systems. Eindhoven, Netherlands: Eindhoven University of Technology, March 2015.

[38] "ECC Report 172: Broadband Wireless Systems Usage in 2300-2400 MHz," March 2012.

[39] ECC, "(CEPT Report 58) Report B2 from CEPT to the European Commission in Response to the Mandate on Harmonised Technical Conditions for the 2300-2400 MHz (2.3 GHz) Frequency Band in the EU for the Provision of Wireless Broadband Electronic Communications Services." July 2015.

[40] "TS 103154 - V1.1.1 - Reconfigurable Radio Systems (RRS); System Requirements for Operation of Mobile Broadband Systems in the 2300 MHz - 2400 MHz Band Under Licensed Shared Access (LSA)," 2014. 